DOI: http://doi.org/10.19175/recom.v11i0.4309

www.ufsi.edu.br/recom

\title{
Práticas de enfermagem relacionadas ao flushing em cateter venoso periférico: estudo descritivo
}

\author{
Nursing practices related to flushing in peripheral venous catheters: a descriptive study \\ Prácticas de enfermería relacionadas com el lavado en catéteres venosos periféricos: estudio \\ descriptivo
}

\section{RESUMO}

Objetivo: Analisar as práticas de enfermagem relacionadas ao flushing para prevenção da obstrução do cateter venoso periférico. Método: Estudo descritivo com aplicação de questionário aos 78 profissionais de enfermagem e checklist durante a observação das práticas de enfermagem numa clínica médica, durante 30 dias. Resultados: $89,7 \%$ dos profissionais de enfermagem realizavam o flushing na prática clínica. A técnica de pushpause era realizada por $12,86 \%$ dos participantes. A seringa mais utilizada foi de $10 \mathrm{ml}$ $(85,72 \%)$, com $10 \mathrm{ml}$ de solução fisiológica $0,9 \%$ (52,86\%). A técnica push-pause foi realizada em $10,7 \%$ das observações e a avaliação da permeabilidade do cateter em 3,6\%. Conclusão: O flushing é utilizado para prevenção da obstrução do cateter nas práticas de enfermagem, entretanto, verificou-se diferenças na técnica, tamanho da seringa, volume de solução fisiológica utilizada e momentos para realização entre os profissionais e o recomendado pela literatura.

Descritores: Enfermagem; Cateterismo Periférico; Obstrução do Cateter

\section{ABSTRACT}

Objective: To analyze nursing practices related to flushing to prevent peripheral venous catheter obstruction. Method: Descriptive study, with application of a semi-structured questionnaire to 78 members of the nursing team, and observation of the clinical practices of medical clinic professionals regarding prevention of obstruction, during 30 days, using a checklist. Results: $89.7 \%$ of nursing professionals performed flushing in clinical practice. The push-pause technique was performed by $12.86 \%$ of the participants. The most used syringe was the $10 \mathrm{ml}(85.72 \%)$ one with $10 \mathrm{ml}$ of $0.9 \%$ saline solution (52.86\%). The push-pause technique was performed in $10.7 \%$ of the observations and catheter permeability evaluation in 3.6\%. Conclusion: Flushing is used to prevent catheter obstruction in nursing practices, however, differences in technique, syringe size, volume of saline solution used, and times of performance were observed among professionals and those recommended by the literature.

Descriptors: Nursing; Catheterization, Peripheral; Catheter Obstruction

\section{RESUMEN}

Objetivo: Analizar las prácticas de enfermería relacionadas con el flushing para prevenir la obstrucción del catéter venoso periférico. Método: Estudio descriptivo con aplicación de cuestionario a 78 profesionales de la enfermería y checklist durante la observación de las prácticas en una clínica médica, durante 30 días. Resultados: El 89,7\% de los profesionales realizó el flushing en la práctica. El 12,86\% de los participantes realizó la técnica de empuje-pausa. La jeringa más utilizada fue la de $10 \mathrm{ml}(85,72 \%)$, con $10 \mathrm{ml}$ de solución salina (52,86\%). La técnica de empuje-pause se realizó en el 10,7\% de las observaciones, y la evaluación de la permeabilidad del catéter en el 3,6\%. Conclusión: El flushing se utiliza para prevenir la obstrucción del catéter en las prácticas de enfermería, sin embargo, se verificaron diferencias en la técnica, el tamaño de la jeringa, el volumen de solución salina y los momentos de realización entre los profesionales y lo recomendado por la literatura.

Descriptores: Enfermería; Cateterismo Periférico; Obstrucción del Catéter
Mirele Herculina Pires ${ }^{1}$

0000-0003-1962-8511

Ana Carolina Almeida ${ }^{2}$

0000-0002-6951-1488

Daniel Camilo Carvalho ${ }^{1}$

0000-0002-8161-3364

Patrícia Oliveira Salgado ${ }^{1}$

0000-0002-0743-0244

Pedro Parreira ${ }^{5}$

0000-0002-3880-6590

Luciene Muniz Braga ${ }^{3}$

0000-0002-2297-395X

${ }^{1}$ Universidade Federal de Viçosa, MG, Brasil.

${ }^{2}$ MILMED Medicina e segurança do trabalho. Vitória, ES, Brasil.

${ }^{3}$ Escola Superior de Enfermagem de Coimbra, Portugal.

Autor correspondente:

Luciene Muniz Braga

E-mail: luciene.muniz@ufv.br

\begin{abstract}
Como citar este artigo:
Pires MH, Almeida AC, Carvalho DC, et al. Práticas de enfermagem relacionadas ao flushing em cateter venoso periférico: estudo descritivo. Revista de Enfermagem do CentroOeste Mineiro. 2021;11:e4309. [Acesso___ ]; Disponível em: DOI: DOI http://doi.org/10.19175/recom.v11i $\underline{0.4309}$
\end{abstract}




\section{INTRODUÇÃO}

A terapia intravenosa é uma das terapêuticas mais utilizadas em ambiente hospitalar e implementada através de um cateter venoso, sendo o cateter venoso periférico (CVP) um dos mais utilizados na prática clínica de enfermagem para administração de medicamentos, hemoderivados, componentes nutricionais e coleta de sangue para fins diagnósticos. No entanto, o tempo de permanência do CVP não excede dois dias na maioria dos casos, sendo removidos devido a complicações, sendo a flebite, a infiltração e a obstrução as mais comuns ${ }^{(1-3)}$. Para a prevenção e minimização dessas complicações é necessário que a equipe esteja treinada para a inserção, vigilância e retirada do CVP, além de documentar e intervir terapeuticamente, ou colaborativamente com o médico, se necessário, em qualquer complicação(4).

A obstrução é uma complicação com taxa de incidência de até $50 \%$. Ela é caracterizada pela incapacidade de coletar sangue, infundir medicamento e realizar a lavagem do CVP, sendo causada por coágulos sanguíneos que se formam pelo retorno de sangue da veia para o lúmen do cateter, por problemas mecânicos do dispositivo e por precipitação de medicamentos incompatíveis no lúmen do cateter ${ }^{(1-2,5-7)}$. A sua ocorrência resulta na interrupção temporária da terapia intravenosa devido à impossibilidade de administrar qualquer medicamento e no risco de infecção e trauma vascular periférico para o paciente, relacionados à remoção e inserção de um novo dispositivo e o sangue coagulado no lúmen do cateter ser um ambiente ideal para o desenvolvimento de microrganismos. Ademais, a inserção de um novo cateter aumenta os custos com materiais, o tempo de assistência de enfermagem, além de causar ansiedade, dor e desconforto para o paciente devido à repunção venosa ${ }^{(2,6-8)}$.

Para prevenir a obstrução e manter a permeabilidade do CVP recomenda-se a lavagem (flushing) do CVP antes e após a administração de cada medicamento, hemoderivados e hemocomponentes, após coleta de amostras de sangue, para a manutenção do cateter ${ }^{(2,5-7)}$. Até recentemente, a solução com heparina era indicada para realizar o flushing no CVP, entretanto, ela é desaconselhada devido ao risco de erro na dosagem, causado pelo processo de diluição e de sua disponibilidade em várias concentrações, e o risco de causar efeitos não desejados, como alergias e sangramentos ${ }^{(8-9)}$. Atualmente, recomenda-se realizar o flushing com solução fisiológica 0,9\% (SF 0,9\%) no CVP, pelo baixo custo, por ser uma solução segura para o paciente, não necessitar de diluição e pela ausência de diferença significativa entre a heparina e a SF 0,9\% em questões de eficácia para manter a permeabilidade dos cateteres venosos ${ }^{(5-10)}$.

Embora existam diretrizes para orientar os cuidados para a manutenção dos $\mathrm{CVP}^{(5)}$, as evidências apontam uma variação nas práticas de enfermagem para prevenção da obstrução, especialmente aquelas acerca do flushing, como volume, frequência e técnica. Na maioria das vezes, essa prática é baseada na política da instituição, na experiência de trabalho pessoal e do colega $^{(2-3,11-12)}$. De acordo com alguns autores, quando não há padronização no cuidado, os riscos de erros e complicações aumentam consideravelmente ${ }^{(11,13)}$.

Apesar de o flushing no CVP com SF 0,9\% ser uma prática simples, de baixo custo, reconhecida por sua eficácia na prevenção da obstrução dos cateteres venosos e contribuir para a qualidade dos cuidados, ainda existem muitas divergências entre os profissionais e falta de protocolos nas instituições devido à escassez de estudos que abordem a eficácia dessa prática. Diante disso, colocou-se a seguinte pergunta de investigação: Quais são as práticas de enfermagem relacionadas ao flushing para prevenção da obstrução do CVP em uma instituição hospitalar de ensino?

Portanto, o presente estudo teve por objetivo analisar as práticas clínicas dos profissionais de enfermagem relacionadas ao flushing para prevenção da obstrução do CVP.

\section{MÉTODO}

Trata-se de um estudo descritivo, realizado em um hospital de ensino de Minas Gerais, Brasil, no período de março a abril de 2019, com os membros da equipe de enfermagem. Os dados foram coletados em dois momentos: primeiro utilizou-se um questionário semiestruturado e no segundo momento a aplicação de um checklist durante a observação dos cuidados realizados pela equipe de enfermagem para prevenção da obstrução no CVP e realização da técnica de flushing no CVP. Os instrumentos foram elaborados pelos autores após revisão integrativa da literatura sobre a temática, sendo incluídas as seguintes evidências: realização do flushing com as técnicas de push-pause e locking, com seringa de 
$10 \mathrm{ml}$ ou maior, com SF 0,9\% e o volume de duas vezes o priming do cateter e suas conexões, e os momentos para realização do flushing (antes, entre e após a administração do último medicamento e hemoderivados ou hemocomponentes; após punção venosa periférica).

Para responder ao questionário foram elegíveis 140 profissionais de enfermagem do hospital estudado. O recrutamento aconteceu no próprio local de trabalho. Foram incluídos enfermeiros, técnicos e auxiliares de enfermagem que desenvolviam atividades assistenciais aos pacientes do hospital em estudo, portanto realizavam o preparo e a administração de medicamentos endovenosos através do CVP. Foram excluídos 62 profissionais, destes, sete estavam de férias, quatro de licença médica, dois de licença maternidade, três estavam afastados por outros motivos, seis por perda, pois não responderam mais de $50 \%$ do questionário e 40 não aceitaram participar do estudo pela assinatura do termo de consentimento livre e esclarecido. Assim, a amostra foi composta por 78 profissionais de enfermagem.

O questionário semiestruturado foi respondido numa sala de reuniões da instituição, no próprio horário de trabalho, num tempo de 10 a 20 minutos e era composto por 20 questões abertas e fechadas, divididas em duas seções: 1 a) questões sobre a experiência e/ou prática clínica dos profissionais quanto a realização do flushing no CVP, no que se refere a realização da técnica, momentos que realizavam o flushing, motivo(s) pelo(s) qual(is) não realizavam, tamanho da seringa utilizada, volume de SF 0,9\% e conhecimento sobre recomendações (inter)nacional sobre o flushing; 2a) caracterização da equipe de enfermagem: idade, sexo, tempo de trabalho na instituição, tempo de exercício profissional, escolaridade e participação em capacitação sobre cateter venoso e flushing.

Num segundo momento, realizou-se durante 30 dias (março a abril de 2019) a aplicação de checklist durante a observação das práticas clínicas dos profissionais de enfermagem, referentes aos cuidados para prevenção da obstrução no CVP e realização da técnica de flushing. A aplicação do checklist iniciava no posto de enfermagem onde era realizado o preparo dos medicamentos, seguindo no quarto do paciente durante a administração dos medicamentos, até o retorno à sala de preparo, em dois principais horários de administração de medicamentos, no diurno às $8 \mathrm{~h}$ e no vespertino às $14 \mathrm{~h}$. Foram incluídos na observação para aplicação do checklist os enfermeiros e técnicos de enfermagem de uma unidade de clínica médica do hospital em estudo e os pacientes com medicamentos administrados através do CVP, e que consentiram sua participação no estudo. Os critérios de exclusão foram outros profissionais de saúde, estudantes de enfermagem, pacientes com cateter venoso central e medicamentos administrados por outras vias exclusivamente. A amostra foi intencional composta por sete técnicos de enfermagem e dois enfermeiros, totalizando 28 observações em diferentes momentos.

O checklist foi aplicado durante o preparo e a administração de medicamentos através do CVP nos pacientes. Foram avaliadas as seguintes variáveis relacionadas com o flushing no CVP: 1) Durante o preparo dos medicamentos: preparação da seringa com SF 0,9\% para realizar o flushing; 2) Durante a administração dos medicamentos: interrupção da solução endovenosa em curso antes da administração de medicamentos; avaliação da permeabilidade do CVP com SF 0,9\%, utilização da técnica push-pause (técnica pulsátil), realização do flushing com pressão contínua no êmbolo da seringa, utilização da solução endovenosa em curso para lavar o cateter após administrar o último medicamento, utilização da técnica de pressão positiva (locking), utilização da seringa de $10 \mathrm{ml}$ para realizar o flushing.

Os dados foram analisados através do Programa Statistical Package for the Social Sciences (SPSS), versão 23.0, através de estatística descritiva (frequência simples e relativa, medidas de tendência central e dispersão). O estudo atendeu às normas dispostas nas Resoluções do Conselho Nacional de Saúde 466/2012 e/ou 510/2016, sendo aprovado pelo comitê de ética e pesquisa com seres humanos (Parecer № 2.965.478; CAAE: 99292918.8.0000.5153).

\section{RESULTADOS}

A idade dos profissionais que responderam ao questionário variou de 20 a 66 anos, sendo 37,5 a média, com maior frequência para a faixa etária de 20 a 40 anos (64,1\%). A maioria era do sexo feminino ( $n=60 ; 76,9 \%)$, técnicos de enfermagem ( $n=67 ; 85,9 \%)$, trabalhavam na instituição há menos de cinco anos ( $n=58 ; 74,4 \%)$. A maioria relatou não ter participado de capacitação sobre cateter venoso $(n=56 ; 71,8 \%)$, no entanto relataram participação em capacitação sobre flushing ( $\mathrm{n}=46 ; 59 \%)$ (Tabela 1). 
Tabela 1 - Caracterização da equipe de enfermagem, Minas Gerais-2019

\begin{tabular}{lc}
\hline Características & $\mathrm{n}(\%)$ \\
\hline Idade (anos) & $50(64,1)$ \\
$20-40$ & $24(30,8)$ \\
$41-60$ & $1(1,3)$ \\
$>60$ & $3(3,8)$ \\
Não responderam & \\
Sexo & $16(20,5)$ \\
Masculino & $60(76,9)$ \\
Feminino & $2(2,6)$ \\
Não responderam & \\
Tempo de exercício profissional na unidade de trabalho & $38(48,7)$ \\
Menos de 1 ano & $20(25,7)$ \\
Entre 2 e 5 anos & $11(14,1)$ \\
Entre 6 e 10 anos & $6(7,7)$ \\
Mais de 10 anos & $3(3,8)$ \\
Não responderam & \\
Tempo de exercício profissional na enfermagem & $10(12,9)$ \\
Menos de 1 ano & $11(14,1)$ \\
Entre 2 e 5 anos & $26(33,3)$ \\
Entre 6 e 10 anos & $28(35,9)$ \\
Mais de 10 anos & $3(3,8)$ \\
Não responderam & \\
Escolaridade & $1(1,3)$ \\
Auxiliar de enfermagem & $67(85,9)$ \\
Técnico de enfermagem & $10(12,8)$ \\
Enfermeiro & $19(24,4)$ \\
Participação em capacitação sobre cateter venoso & $3(3,8)$ \\
Não responderam & $46(59)$ \\
Participação em capacitação sobre flushing & $p e s q u i s a, 2019$. \\
\hline & Fonte: Dados da
\end{tabular}

A maioria dos participantes relatou realizar a técnica de flushing na prática clínica ( $n=70 ; 89,7 \%)$. Em relação à técnica utilizada para realizar 0 flushing, o push-pause era realizada por apenas 12,86\% dos participantes (Figura 1).

Figura 1 - Técnicas utilizadas lavar o cateter venoso periférico, Minas Gerais-2019

Técnicas utilizadas para lavar o cateter venoso periférico

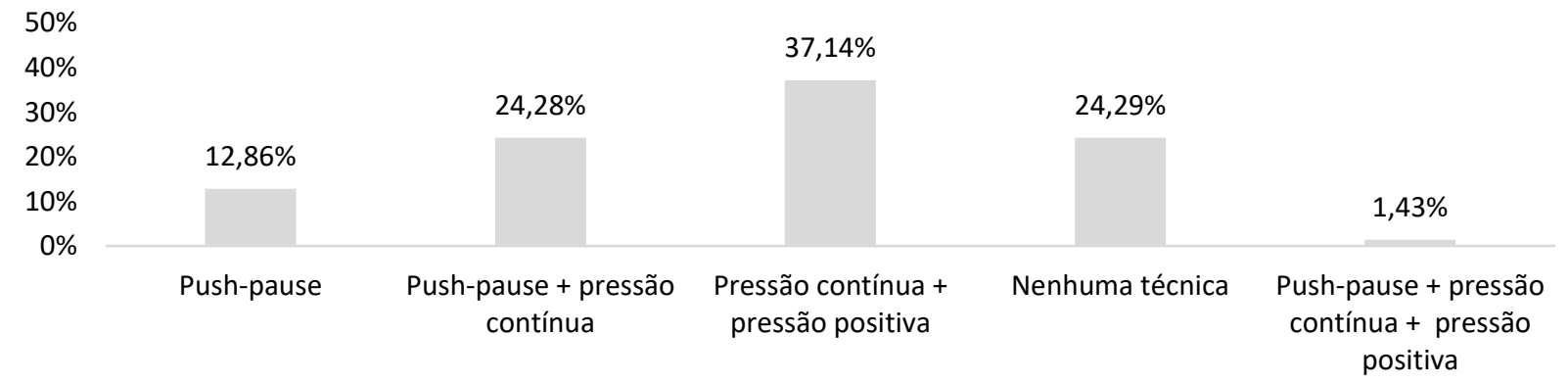

Fonte: Dados da pesquisa, 2019.

Em relação ao momento de realização do flushing, apenas sete profissionais (10\%) realizavam o flushing nos quatro momentos recomendados pela literatura, ou seja, após a inserção do CVP, antes de administrar qualquer medicação, após a administração de cada medicamento e após a administração do último medicamento (Figura 2). 


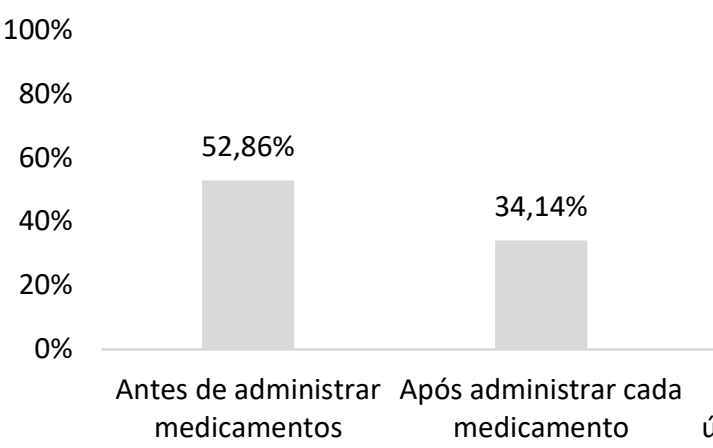

Os motivos pelos quais os oitos profissionais afirmaram não realizar o flushing foram a falta de material disponível na instituição $(n=1 ; 12,5 \%)$, ausência de protocolo $(n=6 ; 75 \%)$ ou outros motivos não descritos ( $n=1 ; 12,5 \%)$.

Quanto ao tamanho da seringa utilizada para a realização do flushing, a maioria dos profissionais afirmou utilizar a seringa de $10 \mathrm{ml}(\mathrm{n}=$ $67 ; 85,89 \%)$. As seringas de $5 \mathrm{ml}(\mathrm{n}=6 ; 7,70 \%)$ e de $20 \mathrm{ml}(\mathrm{n}=5 ; 6,41 \%)$ foram as menos citadas.

A maioria dos participantes utilizava $10 \mathrm{ml}$ de SF 0,9\% para realizar o flushing $(n=41 ; 52,58 \%)$, $34,61 \%$ utilizava $5 \mathrm{ml}(\mathrm{n}=27), 7,69 \%$ utilizava $3 \mathrm{ml}$ $(n=6)$ e $2,56 \%$ utilizava $4 \mathrm{ml}(\mathrm{n}=2)$ ou $20 \mathrm{ml}(\mathrm{n}=2)$. Para manter o CVP preenchido com SF 0,9\%, a maioria dos profissionais utilizava $10 \mathrm{ml}(\mathrm{n}=42$; $53,8 \%), 26,9 \%$ utilizava $5 \mathrm{ml}(\mathrm{n}=21), 6,4 \%$ utilizava $3 \mathrm{ml}(\mathrm{n}=5)$, e os demais participantes $(n=10$; $12,9 \%$ ) não utilizavam, ou outros volumes, ou não responderam.
$78,57 \%$

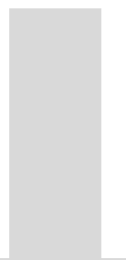

$64,28 \%$

Após administrar o último medicamento
$10,00 \%$

Após inserir o CVP Nos quatro momentos recomendados

Fonte: Dados da pesquisa, 2019.

Tabela 2 - Adesão da equipe de enfermagem às práticas para prevenção da obstrução do cateter venoso periférico, Minas Gerais-2019.

\begin{tabular}{lc}
\hline Práticas de enfermagem para prevenção de obstrução no cateter venoso periférico & $\mathrm{n}(\%)$ \\
\hline Durante o preparo dos medicamentos & $3(10,7)$ \\
Preparação da seringa com solução fisiológica 0,9\% para realizar flushing & $20(71,4)$ \\
Durante a administração dos medicamentos & $1(3,6)$ \\
Interrupção da administração da solução endovenosa em curso & $3(10,7)$ \\
Avaliação da permeabilidade do cateter com solução fisiológica 0,9\% antes da administração do primeiro & $9(32,1)$ \\
medicamento & $3(10,7)$ \\
Utilização da técnica push-pause após o último medicamento & $23(82,1)$ \\
Realização do flushing empurrando o êmbolo da seringa & $8(66,6)$ \\
Utilização da solução endovenosa em curso para lavar o cateter após administrar o último medicamento & \\
Utilização da técnica de locking & Utilização da seringa de 10 ml para realizar flushing
\end{tabular}

Fonte: Dados da pesquisa, 2019

\section{DISCUSSÃO}

Os cateteres venosos podem obstruir quando não há uma vigilância ou cuidados
Apesar de não haver procedimento operacional padrão (POP) na instituição sobre a técnica de flushing para prevenção da obstrução dos cateteres venosos, mais da metade dos profissionais de enfermagem $(n=53 ; 68 \%)$ afirmou conhecer alguma recomendação (inter)nacional sobre oflushing, 26,9\% dos profissionais relataram não conhecer nenhuma recomendação $(n=21)$ e o restante dos participantes $(n=4 ; 5,1 \%)$ não respondeu à essa pergunta.

Durante a aplicação do checklist verificou-se o flushing sendo realizado com a técnica pushpause em três observações (10,7\%). A avaliação da permeabilidade do CVP antes da administração do primeiro medicamento foi observada uma vez $(3,6 \%)$. A maioria dos profissionais utilizavam seringa de $10 \mathrm{ml}$ para realizar o flushing e com pressão positiva, técnica de locking (82,1\%) (Tabela 2). adequados destinados a eles. A obstrução também pode ocorrer quando os cateteres não são mantidos preenchidos, quando não estão em uso 
ou quando não se realiza o flushing ${ }^{(5)}$. O flushing, enquanto método de prevenção de obstrução, aumenta o tempo de viabilidade do cateter, o que gera vantagens significativas para o paciente e para o cuidado prestado ${ }^{(7,14)}$. No presente estudo, a maioria dos profissionais relatou realizar o flushing $(89,7 \%)$, sendo um aspecto positivo para a prevenção dessa complicação. Esse achado vai de encontro a outro estudo transversal realizado recentemente com profissionais de enfermagem do Brasil e de Portugal, cuja taxa de realização de flushing era $84,2 \%{ }^{(13)}$.

Apenas $12,86 \%$ dos profissionais de enfermagem afirmaram utilizar a técnica pushpause, técnica indicada para realizar o flushing ${ }^{(5)}$. De acordo com o questionário $(37,14 \%)$ e o checklist na observação das práticas $(32,1 \%)$, a maioria dos profissionais utilizava pressão contínua no êmbolo da seringa (fluxo laminar), mantendo pressão positiva ao término da administração do medicamento. Um estudo de simulação experimental realizado sobre a lavagem do cateter venoso revelou que a eficiência da lavagem dos cateteres está diretamente relacionada com o tipo de fluxo realizado, logo, o fluxo intermitente (pushpause) permite a remoção de fluidos no lúmen do cateter em menor tempo e com maior eficácia quando comparado ao fluxo contínuo/laminar (pressão contínua no êmbolo da seringa)(15).

Para realizar a lavagem do CVP e prevenir a obstrução é fundamental realizar o flushing com a técnica intermitente (push-pause), pois ela aumenta a eficácia da limpeza do lúmen do cateter devido a maior força de cisalhamento ${ }^{(16-18)}$. Tratase de uma técnica simples e de baixo custo, sendo eficiente, também, na prevenção de bacteremia no cateter, pois evita acúmulo de sangue no lúmen do cateter e proliferação de microrganismos ${ }^{(19)}$.

Nesse estudo, 38,57\% dos participantes afirmaram realizar a técnica de locking (pressão positiva), sendo confirmado esse achado nos dados do checklist em 32,1\% das observações das práticas clínicas. A técnica de locking, consiste em fechar a conexão do sistema de infusão (conexões dupla via ou torneira de três vias, por exemplo) ligada ao cateter simultaneamente à administração do volume final da solução do flushing, em torno de 0,5ml. Essa ação mantêm pressão positiva com a coluna de SF $0,9 \%$ dentro do lúmens do cateter prevenindo o retorno de sangue para o lúmen e, consequente obstrução caso não seja realizado o flushing imediatamente ${ }^{(5-}$ 6).
No presente estudo, durante a observação das práticas, verificou-se que a maioria dos profissionais realizava o flushing somente após a administração do último medicamento, e apenas $10 \%$ o realizava nos quatro momentos recomendados pela literatura, ou seja, após a inserção do CVP a fim de remover o sangue presente no lúmen do cateter durante a punção venosa, para avaliar a permeabilidade do cateter e conferir se o mesmo se encontra posicionado corretamente na veia do paciente; antes da administração de qualquer medicação para avaliar a permeabilidade do cateter; após a administração de cada uma das medicações administradas no cateter, com o intuito de evitar a interação de medicamentos incompatíveis; após a administração do último medicamento a fim de remover os resíduos de medicamento do lúmen do cateter e assegurar a administração completa da dose prescrita ${ }^{(5,20)}$; após a administração de hemocomponentes, após a coleta de sangue e a cada 8 a 12 horas quando o CVP não estiver em uso $^{(5,16)}$. Segundo Goossens ${ }^{(6)}$ e a Infusion Nurses Society ${ }^{(5)}$, o flushing com SF $0,9 \%$ deve ser realizado antes de administrar o primeiro medicamento no CVP do paciente com o objetivo de avaliar a permeabilidade do cateter, em seguida realizar a administração do medicamento e novamente realizar o flushing com SF 0,9\% para remover os resíduos do medicamento do lúmen do cateter e evitar incompatibilidade entre os medicamentos ou soluções.

Em relação ao tamanho da seringa, o resultado do presente estudo corrobora com o que é preconizado pela literatura, ou seja, usar seringas de maior calibre, $10 \mathrm{ml}$ ou mais, pois melhora o resultado da lavagem do cateter e causa menores danos à veia por apresentar uma baixa pressão ${ }^{(5,16,21)}$. Seringas pré-preenchidas de $10 \mathrm{ml}$ utilizadas no flushing, aumentam a permeabilidade ${ }^{(11)}$ e diminuem o risco de danos ao cateter, e consequente ruptura na veia do paciente ${ }^{(6)}$. As seringas pré-preenchidas apresentam o mesmo objetivo das seringas preparadas manualmente pelo profissional de enfermagem, no entanto as primeiras proporcionam menor manipulação, menor tempo no preparo e diminuem o risco de contaminação do dispositivo ou da SF $0,9 \%{ }^{(5)}$, pois são prépreparadas pela indústria farmacêutica dentro de condições assépticas controladas, como capela de fluxo laminar e processo de esterilização.

No presente estudo, os profissionais não levaram em consideração o priming do CVP 
(volume do lúmen) e de sua extensão para administrar a SF $0,9 \%$, uma vez que os volumes mais utilizados foram $10 \mathrm{ml}(33,3 \%)$ e $5 \mathrm{ml}(52,6 \%)$, semelhante a outros estudos ${ }^{(2,7,11)}$, contrariando a recomendação da Infusion Nurses Society ${ }^{(5)}$ de utilizar o dobro do volume interno do cateter e de suas extensões acessórias. No entanto, cabe mencionar que é fundamental, que o profissional de enfermagem ao escolher o volume de solução, levar em consideração além do volume do lúmen do cateter (priming) e suas extensões (extensão de dupla via, por exemplo), a idade e restrições hídricas do paciente, e as características dos medicamentos a serem administrados, uma vez que após a infusão de soluções lipídicas e hemocomponentes, por exemplo, podem exigir um volume maior de SF 0,9\% para a limpeza do lúmen do cateter, normalmente $20 \mathrm{ml} \mathrm{l}^{(5)}$, assim como após o refluxo de sangue no lúmen do CVP e suas conexões devido a punção venosa ou por outro motivo.

A Infusion Nurses Society ${ }^{(5)}$ recomenda realizar o flushing com um volume de SF 0,9\% duas vezes o volume do cateter e do dispositivo complementar (priming), que pode ser um tubo extensor ou torneira de três vias, por exemplo. Essa quantidade, geralmente, varia de $1 \mathrm{ml}$ a $3 \mathrm{ml}$ em um CVP, a depender do tipo de dispositivo complementar utilizado. Volumes maiores, de $5 \mathrm{ml}$ para o CVP e $10 \mathrm{ml}$ para o cateter venoso central são indicados para diminuir o acúmulo de fibrina, medicamentos precipitados ou outras substâncias no lúmen do cateter. Para Goossens ${ }^{(6)}$ o volume de solução para realizar o flushing em CVP pode ser de $5 \mathrm{ml}$, pelo fato de o CVP ter um pequeno calibre.

Quanto ao momento de realização do flushing, tamanho da seringa e quantidade de SF $0,9 \%$, os resultados são semelhantes aos encontrados na literatura, com volume de solução e seringas de $2 \mathrm{ml}, 3 \mathrm{ml}, 5 \mathrm{ml}$ e $10 \mathrm{ml} \mathrm{l}^{(2,7,11,13)}$.

Apesar dos profissionais responderem ter participado de curso de capacitação sobre flushing (59\%), parece que esses conhecimentos não foram implementados na prática, pois na observação das práticas, verificou-se que a técnica de push-pause foi realizada em apenas $10,7 \%$ das observações, o uso da seringa de $10 \mathrm{ml}$ em $66,6 \%$ e a avaliação da permeabilidade do cateter com SF0,9\% antes da administração do primeiro medicamento em 3,6\% das observações. Essa ausência de cuidado qualificado pode ser devido à falta de protocolo institucional (75\%) sobre técnica de flushing para prevenção da obstrução dos cateteres venosos e por consequente a ausência de material disponível
$(12,5 \%)$ conforme evidenciado pelos resultados do questionário. Apesar das melhores evidências disponíveis reconhecerem e recomendarem o uso do flushing nas práticas para prevenção da obstrução nos cateteres venosos ${ }^{(5-6,15-17)}$, não se verifica uma transferência desse conhecimento para as práticas clínicas ${ }^{(2,11-13,22)}$. Tal fato pode ser devido à ausência de evidência forte e variabilidade clínica sobre o volume, frequência e técnica de flushing, conforme documentado nesse estudo em outros ${ }^{(2,7,11,13,17)}$, devido ao dimensionamento de profissionais de enfermagem insuficientes em relação ao grau de dependência dos pacientes e volume de trabalho, resultando na falta de tempo do profissional para realizar um cuidado qualificado, além da ausência de conhecimento das melhores evidências para reconhecer o flushing como um cuidado prioritário, que impacta nos custos assistenciais, no tempo de assistência de enfermagem, na qualidade dos cuidados e segurança do paciente $e^{(2,11)}$.

\section{CONCLUSÃO}

O flushing é uma prática de enfermagem realizada pela maioria dos profissionais. No entanto, a falta de materiais para implementar a técnica e de protocolo institucional foram citados como fatores dificultadores para a adesão à prática, resultando em divergência nas práticas quanto ao momento de realização do flushing, à técnica utilizada, ao tamanho da seringa e quantidade de SF 0,9\%.

O presente estudo contribuiu para a reflexão da prática clínica de enfermagem ao revelar a necessidade de os serviços de enfermagem atualizarem, padronizarem e divulgarem seus protocolos sobre o flushing e implementar capacitações para os profissionais de enfermagem. No ensino, reforça a necessidade de o flushing e outros conteúdos relacionados à terapia intravenosa serem incluídos nas ementas das disciplinas básicas dos cursos de enfermagem com foco na prevenção, segurança do paciente e qualidade dos cuidados. Além disso, apresenta contribuições para a pesquisa, indicando áreas que necessitam de estudos, como análise da eficácia da técnica de flushing, dos diferentes tamanhos de seringas, dos diferentes volumes de solução fisiológica e da técnica push-pause, além de apontar a necessidade de analisar as práticas de flushing em outras realidades clínicas de atuação da enfermagem. 
Têm-se como principais limitações ser um estudo descritivo, com pequena amostra de participantes e de conveniência, o número reduzido de observações, os dados serem de uma única instituição, não permitindo a generalização dos resultados e ausência de análise dos fatores de risco para a ocorrência de obstrução tendo em vista as diferentes práticas adotas em relação à técnica de flushing, os volumes de SF 0,9\% e tamanho de seringa utilizados.

\section{REFERÊNCIAS}

1. Danski MTR, Johann DA, Vayego SA, Oliveira GRL, Lind J. Complications related to the use of peripheral venous catheters: a randomized clinical trial. Acta Paul. Enferm. 2016;29(1):84-92. DOI: 10.1590/1982-0194201600012.

2. Braga LM, Parreira PM dos SD, Arreguy-Sena C, Carlos DM, Mónico L dos SM, Henriques MAP. Incidence rate and the use of flushing in the prevention of obstructions of the peripheral venous catheter. Texto e Contexto Enferm. 2018;27(4):e2810017. DOI: 10.1590/0104$\underline{07072018002810017 .}$

3. Parreira P, Sousa LB, Marques IA, Santos-Costa $P$, Braga LM, Cruz A, et al. Double-chamber syringe versus classic syringes for peripheral intravenous drug administration and catheter flushing: a study protocol for a randomised controlled trial. Trials. 2020;21(1):78. DOI: 10.1186/s13063-019-3887-1.

4. Johann DA, Danski MTR, Vayego SA, Barbosa DA, Lind J. Risk factors for complications in peripheral intravenous catheters in adults: Secondary analysis of a randomized controlled trial. Rev. latinoam. enferm. 2016;24:e2833. DOI: 10.1590/15188345.1457.2833.

5. Gorski LA, Hadaway LM, Hagle ME, Broadhurst $D$, Clare SM, Kleidon $T$, et al. Infusion Therapy Standards of Practice, 8th Edition. J Infus Nurs.2021;44(1S Suppl 1):S1-S224. DOI: 10.1097/NAN.0000000000000396.

6. Goossens GA. Flushing and locking of venous catheters: available evidence and evidence deficit. Nurs Res Pract. 2015;2015:12. DOI: 10.1155/2015/985686.

7. Keogh S, Flynn J, Marsh N, Mihala G, Davies K, Rickard C. Varied flushing frequency and volume to prevent peripheral intravenous catheter failure: a pilot, factorial randomised controlled trial in adult medical-surgical hospital patients. Trials. 2016;17:348. DOI: 10.1186/s13063-016-1470-6.

8. Alexandrou E, Ray-Barruel G, Carr PJ, Frost SA, Inwood S, Higgins N, et al. Use of short peripheral intravenous catheters: characteristics, management, and outcomes worldwide. J Hosp Med. 2018;13(5):E1-E7. DOI: 10.12788/jhm.3039.

9. Xu L, Hu Y, Huang X, Fu J, Zhang J. Heparinized saline versus normal saline for maintaining peripheral venous catheter patency in China: an open-label, randomized controlled study. J Int Med Res. 2017;45(2):471-80. DOI: 10.1177/0300060516685203.

10. Sotnikova C, Fasoi G, Efstathiou F, Kaba E, Bourazani $M$, Kelesi $M$. The efficacy of normal saline (N/S 0.9\%) versus heparin solution in maintaining patency of peripheral venous catheter and avoiding complications: a systematic review. Mater Sociomed. 2020;32(1):29-34. DOI: 10.5455/msm.2020.32.29-34.

11. Norton $C B$, Lauria HA, Hauser $P$, Smith-miller CA, Baker D. Nurses' short peripheral catheter flushing practices: implications for patient care, nursing education, and policy. I Infus Nurs. 2019;42(5):228-36.

DOI:

10.1097/NAN.0000000000000337.

12. Parreira $P$, Marques IA, Santos-Costa $P$, Sousa LB, Braga L, Apóstolo J, et al. Peripheral intravenous catheter flushing: a scoping review protocol. Rev. Enf. Ref. 2020.serV(1):e19066. DOI: 10.12707/RIV19066.

13. Parreira $P$, Vicente $R$, Bernardes RA, Sousa LB, Serambeque $B$, Costa $P$, et al. The flushing procedure in nursing practices: a cross-sectional study with Portuguese and Brazilian nurses. Heliyon. 2020;6(8):e04579. DOI: 10.1016/j. heliyon. 2020.e04579.

14. Lanza VE, Alves APP, Camargo AMS, Cacciari P, Montandon DS, Godoy S de. Preventive measures of infection related to peripheral venous catheter: adherence in intensive care. Rev. RENE. 2019;20(1):e40715. DOI: $10.15253 / 2175-$ $\underline{6783.20192040715 .}$

15. Vigier JP, Merckx J, Coquin JY, Flaud P, Guiffant $G$. The use of a hydrodynamic bench for experimental simulation of flushing venous catheters: Impact on the technique. ITBM-RBM. 
2005;26(2):147-9.

DOI: 10.1016/j.rbmret.2005.03.001.

16. Royon L, Durussel JJ, Merckx J, Flaud P, Vigier JP, Guiffant $G$. The fouling and cleaning of venous catheters: a possible optimization of the process using intermittent flushing. Chem Eng Res Des. 2012;90(6):803-7.

DOI: 10.1016/i.cherd.2011.10.004.

17. Guiffant G, Durussel JJ, Merckx J, Flaud P, Vigier $J P$, Mousset P. Flushing of intravascular access devices (IVADS) - Efficacy of Pulsed and Continuous Infusions. J Vasc Access. 2012;13(1):75-8. DOI: 10.5301/JVA.2011.8487.

18. Zhu L, Liu H, Wang R, Yu Y, Zheng F, Yin J. Mechanism of pulsatile flushing technique for saline injection via a peripheral intravenous catheter. Clinical Biomechanics (Bristol). 2020;80:105103-1051103. DOI: 10.1016/j.clinbiomech.2020.105103.

19. Ferroni A, Gaudin F, Flaud P, Durussel J-J, Descamps $P$, Berche $P$, et al. Pulsative flushing as a strategy to prevent bacterial colonization of vascular access devices. Med Devices. 2014;7:37983. DOI: $10.2147 /$ MDER.S71217.

20. Deparment of health. Guideline - Guideline peripheral intravenous catheter (PIVC). [citado em: 05 de mar. 2021];2016;1-26. Disponível em: https://www.health.qld.gov.au/ data/assets/pdf file/0025/444490/icare-pivc-guideline.pdf.

21. Keogh, S., Marsh, N., Higgins, N., Davies, K., Rickard, C. A time and motion study of peripheral venous catheter flushing practice using manually prepared and prefilled flush syringes. J Infus Nurs. 2014;37(2):96-101. DOI: 10.1097/NAN.00000000000000024.

22. Oliveira ASS, Costa PJS, Fraveto JMGN, Costa FJG, Osório NIA Cosme ASTC, et al. Nurses' peripheral intravenous catheter-related practices: a descriptive study. Rev. Enf. Ref. 2019;111-22. DOI: https://doi.org/10.12707/RIV19006.
Editores Responsáveis:

Patrícia Pinto Braga

Kellen Rosa Coelho Sbampato

Nota: Pesquisa financiada pelo CNPq- Bolsa de iniciação científica PIBIC/CNPq 2018-2019.

Recebido em: 21/04/2021

Aprovado em: 19/08/2021 